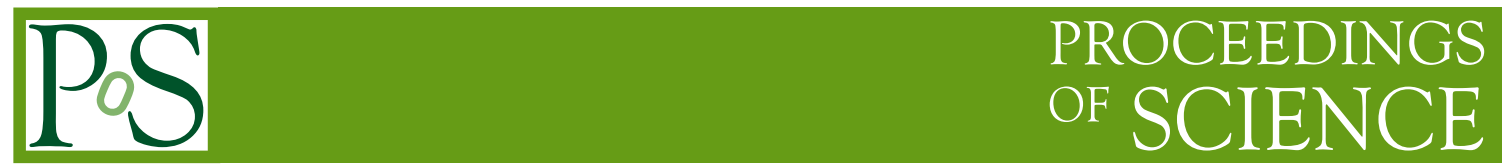

\title{
pMSSM combination of SUSY searches at the LHC
}

\author{
Alberto Cervelli* on behalf of ATLAS and CMS collaboration \\ Universität Bern \\ E-mail: alberto.cervelli@lhep.unibe.ch
}

\begin{abstract}
The results of supersymmetric searches at the LHC are usually presented in the context of simplified models, with a single specific production channel and decay mode for the supersymmetric particles. In full SUSY models, several production and decay channels are expected, and the limits on supersymmetric particle masses might be weaker. In the following we will presents the combination of the results from each of the ATLAS and CMS collaborations to place constraints on the 19-parameter phenomenological MSSM (pMSSM).
\end{abstract}

The European Physical Society Conference on High Energy Physics 22-29 July 2015

Vienna, Austria

${ }^{*}$ Speaker. 


\section{Introduction}

Two of the major highlights in LHC Run 1 by ATLAS [1] and CMS [2] are the discovery of the Higgs boson [3, 4], and the lack of observation of any new physics in the observed energy range. In particular, no evidence for SUSY was found: searches for SUSY are made in the context of, mostly, model independent searches, achieved by using simplified model (SMS) approaches[5], or a Constrained Minimal Supersymmetric Model (CMSSM). The latter uses only a few parameters, it can't reproduce the full mass spectra predicted by SUSY and hence may over-constrain the mass spectra. While the former may reproduce the full spectrum, it does not account for the processes leading to the final states.

In order to have a better understanding of the underlaying physics in the sought for processes and complement these searches, a generic model dependent search is crucial. One of the best models to use is the phenomenological supersymmetry model (pMSSM)[6], which covers a wide range of final state topologies. This interpretation is complimentary to SMS in the sense that parameter space points excluded by SMS searches may still be accessible in pMSSM interpretation and vice versa.

pMSSM model reduces the number of SUSY parameters from the 120 of the minimal supersymmetric model (MSSM) to 19 parameters: this reduction is achieved by assuming, as phenomenological observations suggest, that in the SUSY sector there are no new sources of CPviolation, there are no FCNC, and that the $1^{s t}$ and $2^{\text {nd }}$ generations satisfy universality. By applying these assumptions, the 19 free parameters are: the three gaugino masses (bino, wino, gluino) $M_{1}, M_{2}, M_{3}$; the ratio of the Higgs doublet VEV, $\tan \beta$; the higgsino mass parameter $\mu$, and the pseudo-scalar Higgs mass $m_{A} ; 51^{\text {st }}$ and $2^{\text {nd }}$ generation sfermion (degenerate masses), and $51^{3 d}$ generation sfermion masses; three trilinear couplings $A_{t}, A_{b}, A_{\tau}$. The higgs doublet and Bino parameters are determined through EWK symmetry breaking, and the lightest neutralino $\tilde{\chi}_{1}^{0}$, is stable and is the lightest SUSY particle (LSP).

To achieve a better sensitivity to pMMSM processes, both ATLAS and CMS performed statistical combinations of the results from the searches in different exclusive final states. In the following we will report about the methods used to obtain such combinations and the impact of the results on the constraining of the pMSSM parameters.

\section{ATLAS Results}

The ATLAS collaboration pursued a large number of searches for different final state signatures in the SUSY framework and these analyses have been individually reinterpreted for pMSSM. To further extend the reach of sensitivity to new physics, ATLAS pursued a statistical combination effort of such searches. In order to achieve this final states produced by similar production processes have been combined. For the statistical combination the individual analysis expected background and signal yields are summed up, while the systematic uncertainties are treated as correlated among the single analyses when arising from the same source (i.e. Jet Energy Scale). With this approach the sensitivity is extended mainly by increasing the statistics of the sample under study. To achieve this result the selections of the analyses to be combined need to be orthogonal to each other, to avoid event double counting. When two (or more) signal selections are not orthogo- 
nal, the best exclusion result among the analysis is considered. Exclusion limits are extracted from the combined analysis, with summed yields and correlated systematics, using the $C L_{s}$ method[7]: the results are presented in 2-Dimensional slices of the pMSSM parameter space, and all other parameters are considered set or decoupled.

ATLAS performed two combinations in searches for electroweakly produced SUSY particles: the searches for 2 and 3 lepton final states have been combined, considering both the case of decoupled sleptons and right-handed sleptons. ATLAS performed a scan of the $\mu-M_{2}$ plane, for varying $\tilde{\ell}$ masses[8]. The results are presented in Fig. 1.

A combination of four different processes mediated by $\tilde{\tau}$, with $2 \tau \mathrm{s}$ in the final states, was made, and it excluded light $\tilde{\chi^{+}}$and $\chi_{1}^{0}$ masses in the range of $150-350 \mathrm{GeV}$ [9], as shown in Fig. 1. The scan was performed for different $\tilde{\tau}$ masses, and it should be noted that for high $M_{2}$ values,

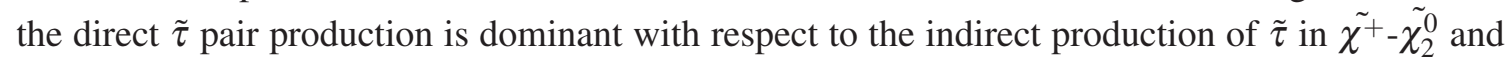

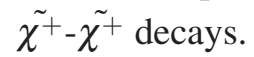
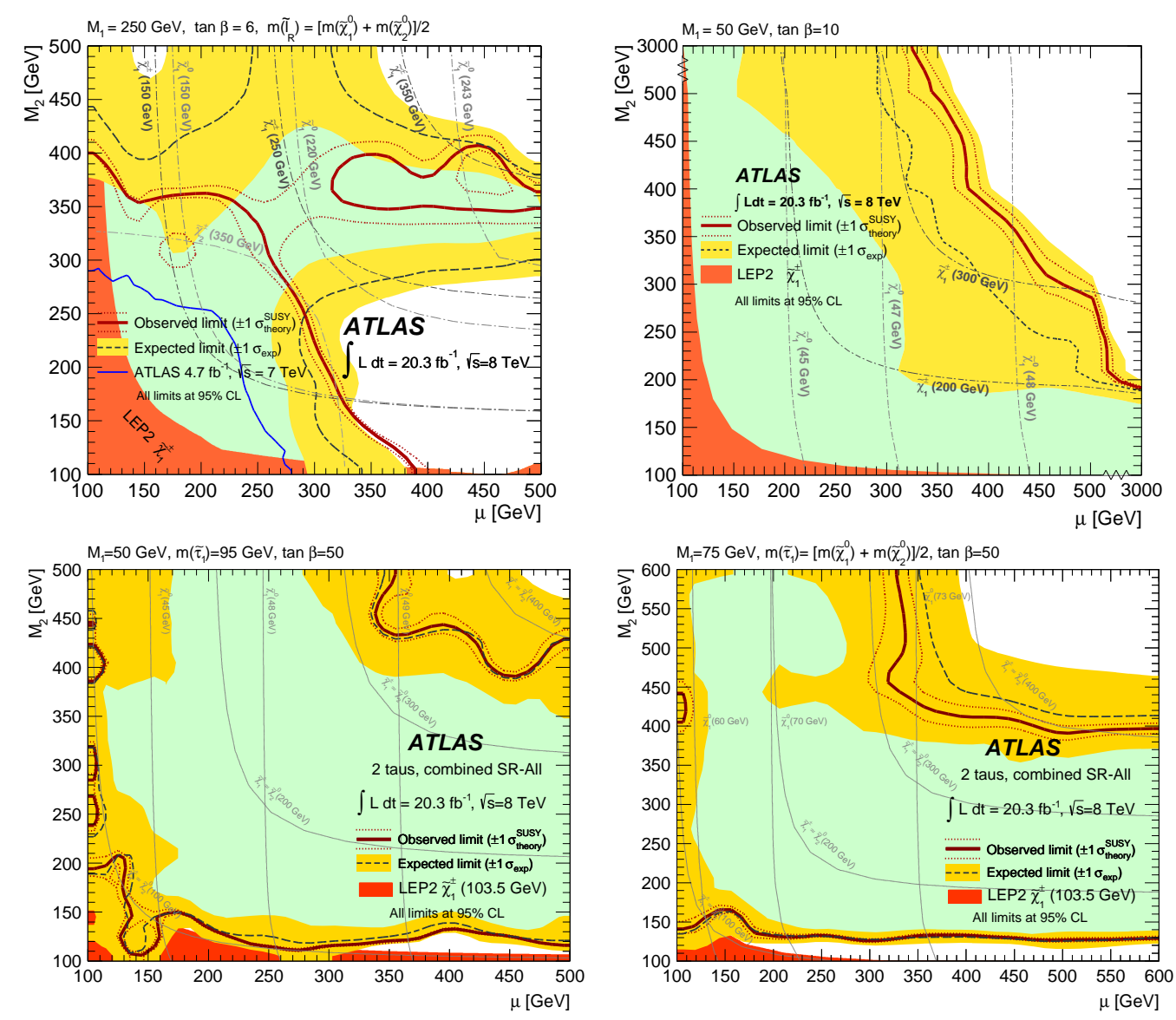

Figure 1: On top row: 95\% CL exclusion for combination of 2 and 3 lepton final states, in $\tilde{\ell}$ mediated scenario for $m(\tilde{\ell})=\left[\tilde{\chi_{1}^{0}}+\tilde{\chi_{2}^{0}}\right] / 2$ (left), and decoupled $\tilde{\ell}$ right [8]. On bottom row: $95 \%$ $\mathrm{CL}$ exclusion for combination of $2 \tau$ final states for $m(\tilde{\tau})=95 \mathrm{GeV}$ (left) and $m(\tilde{\tau})=\left[\tilde{\chi}_{1}^{0}+\tilde{\chi}_{2}^{0}\right] / 2$ (right) [9].

In the framework of $3^{r d}$ generation SUSY particles searches, combinations helped in extend- 
ing the sensitivity for $\tilde{t}$ and $\tilde{b}$ production. ATLAS searched for a $\tilde{t}-\tilde{b}$-higgsino triplet model in a naturalness inspired pMSSM scenario, defined by $m(\tilde{t})<1 \mathrm{TeV}$, low $\mu$, and $m\left(\tilde{\chi}_{1}^{0}\right), m\left(\tilde{\chi}_{2}^{0}\right)$ of $O(\mu)$. The large number of light particles allows many decay chains, so ATLAS combined the results from $t+0$ lepton, $t+1$ lepton, $t+b, t+Z+2 t, g+3 b$, and $t+c$ searches [10]. The results, reported in Fig.2, are obtained by selecting the best exclusion limit among the combined analyses.

A slightly different approach was used in $\tilde{t}+1 \ell$ searches [11]: in this case 27 different pMSSM models were looked for, by varying the masses of $\tilde{t}$, charginos, and neutralinos, as well as the branching ratios of $\tilde{t}$ to lighter particles. The results of the searches are presented as CL exclusion as a function of the branching ratios of $\tilde{t} \rightarrow \tilde{b} \tilde{\chi}^{+}$and $\tilde{t} \rightarrow \tilde{t} \tilde{\chi}_{1}^{0}$. Results are reported in Fig.2.
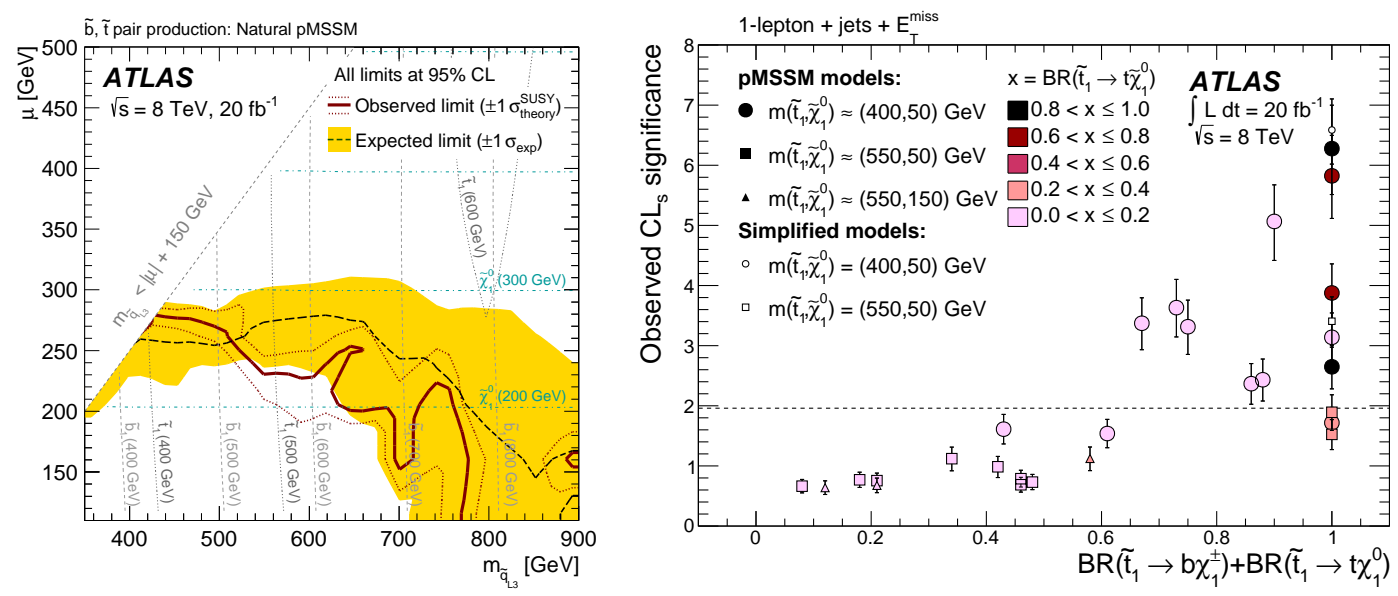

Figure 2: On the left: Expected and observed 95\% CL exclusion limits for the naturalness-inspired set of pMSSM models from the combination $\mathrm{t} 0 \mathrm{~L}, \mathrm{t} 1 \mathrm{~L}$ and tb analyses using the signal region yielding the smallest CLs [10]. On the right: observed CLs significance values for the 27 pMSSM: points above the dashed line, indicating the CLs significance corresponding to $95 \% \mathrm{CL}$, are excluded [11].

\section{CMS results}

CMS result combination pursued a Bayesian treatment of statistical data, making a global fit of the 19 free pMSSM parameters [12]: a prior of non CMS results ( $p^{\text {non-DCS }}$ ) is built from existing results, not coming from CMS SUSY searches, then a posterior probability is built using the SUSY searches results as $p\left(\theta \mid D^{\mathrm{CMS}}\right)=L\left(D^{\mathrm{CMS}} \mid \theta\right) p^{\text {non-DCS }}(\theta)$, this posterior function describes the pdf of each parameter. To assess the statistical significance of the search, the $\mathrm{Z}$ score is used, defined as $Z=\operatorname{sign}\left(\ln B_{10} \sqrt{2\left|\ln B_{10}\right|}\right)$, where $B_{10}(\theta)=L\left(D^{\mathrm{CMS}} \mid \theta, H_{1}\right) / L\left(D^{\mathrm{CMS}} \mid \theta, H_{0}\right)$, and $H_{0}, H_{1}$ are the background and signal hypothesis. The $\mathrm{Z}$ score can be considered equivalent to the frequentist sigma: $\mathrm{Z}>0$ means observation, $\mathrm{Z}<0$ means exclusion, while a $\mathrm{Z}$ value around 0 means that the search is not sensitive to the particular parameter or model.

CMS combined three broad ranges of searches, and both the 7 an $8 \mathrm{TeV}$ data samples, the combined final states are divided in:

- HT+MHT: more than 3 jets and missing transverse energy, targeting gluino and squark production with long hadronic cascades; 
Table 1: Fractions of phase space with $\mathrm{Z}$ scores values below $-1.64,-3$, and -5 . Between brackets is shown the fraction of parameter space for which the particular analysis is the only one under that $\mathrm{Z}$ score value. Taken from [12].

\begin{tabular}{|l|cc|cc|cc|}
\hline & $\mathrm{p}(\mathrm{Z}<-1.64)$ & \multicolumn{2}{c|}{$\mathrm{p}(\mathrm{Z}<-3)$} & $\mathrm{p}(\mathrm{Z}<-3)$ \\
\hline \multicolumn{7}{|c|}{$7 \mathrm{TeV}$} \\
\hline HT+MHT & 0.40 & $(0.23)$ & 0.26 & $(0.15)$ & 0.17 & $(0.09)$ \\
HT+MET + b-jets & 0.19 & $(0.02)$ & 0.14 & $(0.02)$ & 0.098 & $(0.02)$ \\
EW production & 0.026 & $(0.008)$ & 0.011 & $(0.005)$ & 0.005 & $(0.002)$ \\
combination & \multicolumn{7}{|c|}{0.42} & \multicolumn{2}{c|}{0.29} & \multicolumn{2}{c|}{0.19} \\
\hline \multicolumn{7}{|c|}{$8 \mathrm{TeV}$} \\
\hline HT+MHT & 0.45 & $(0.16)$ & 0.37 & $(0.16)$ & 0.30 & $(0.14)$ \\
HT+MET + b-jets & 0.31 & $(0.03)$ & 0.23 & $(0.02)$ & 0.17 & $(0.02)$ \\
EW production & 0.050 & $(0.019)$ & 0.029 & $(0.013)$ & 0.016 & $(0.008)$ \\
combination & 0.47 & \multicolumn{2}{c|}{0.41} & 0.32 \\
\hline \multicolumn{7}{|c|}{$7+8 \mathrm{TeV}$} \\
\hline HT+MHT & 0.48 & $(0.16)$ & 0.40 & $(0.16)$ & 0.32 & $(0.14)$ \\
HT+MET + b-jets & 0.34 & $(0.03)$ & 0.26 & $(0.02)$ & 0.19 & $(0.02)$ \\
EW production & 0.055 & $(0.017)$ & 0.032 & $(0.012)$ & 0.018 & $(0.007)$ \\
combination & 0.51 & \multicolumn{2}{c|}{0.44} & 0.34 \\
\hline
\end{tabular}

- HT+MET+b-jets: at least 3 jets, of which at least $1 \mathrm{~b}$ jet, focused on gluinos decaying to $3^{\text {rd }}$ generation particles;

- EW production: leptons and missing transverse energy targets neutralino, chargino, and slepton pair production.

The $L\left(D^{\mathrm{CMS}}\right)$ function may be factorized only if the selection of the different analyses are not overlapping. In case the signal region defined in a single analysis are overlapping, the best performing one is used to build the combined likelihood function (i.e. for HT+MET+b-jets at $7 \mathrm{TeV}$ ); in case two different analysis present overlapping datasets, their likelihoods can not be factorized, so the individual $Z$ scores of the two analyses are combined independently, by adding them in quadrature.

CMS measured the $\mathrm{Z}$ scores for the 3 analyses independently and for the combination, a scan of many points in the 19-dimensional space of pMSSM parameters is performed. The fraction of excluded models for each analysis and for the combinations is reported in Tab.1, and the $\mathrm{Z}$ score distributions are reported in Fig.3. This approach allows also to have $\mathrm{Z}$ distributions for each single parameter, assigning a likelihood, and hence a $\mathrm{Z}$ score to each one of the scanned values. A full report can be found in [12].

\section{Conclusion}

LHC Run 1 has been a very successful experience for both ATLAS and CMS, however no evidence of physics beyond the standard model was found. Many searches were performed by both collaborations aiming at SUSY discovery. The combination of the single-analysis results in each experiment proved to be a very solid and useful method to shed even more light on the SUSY parameter space. In particular, the pMSSM interpretation profited most from this approach, which 

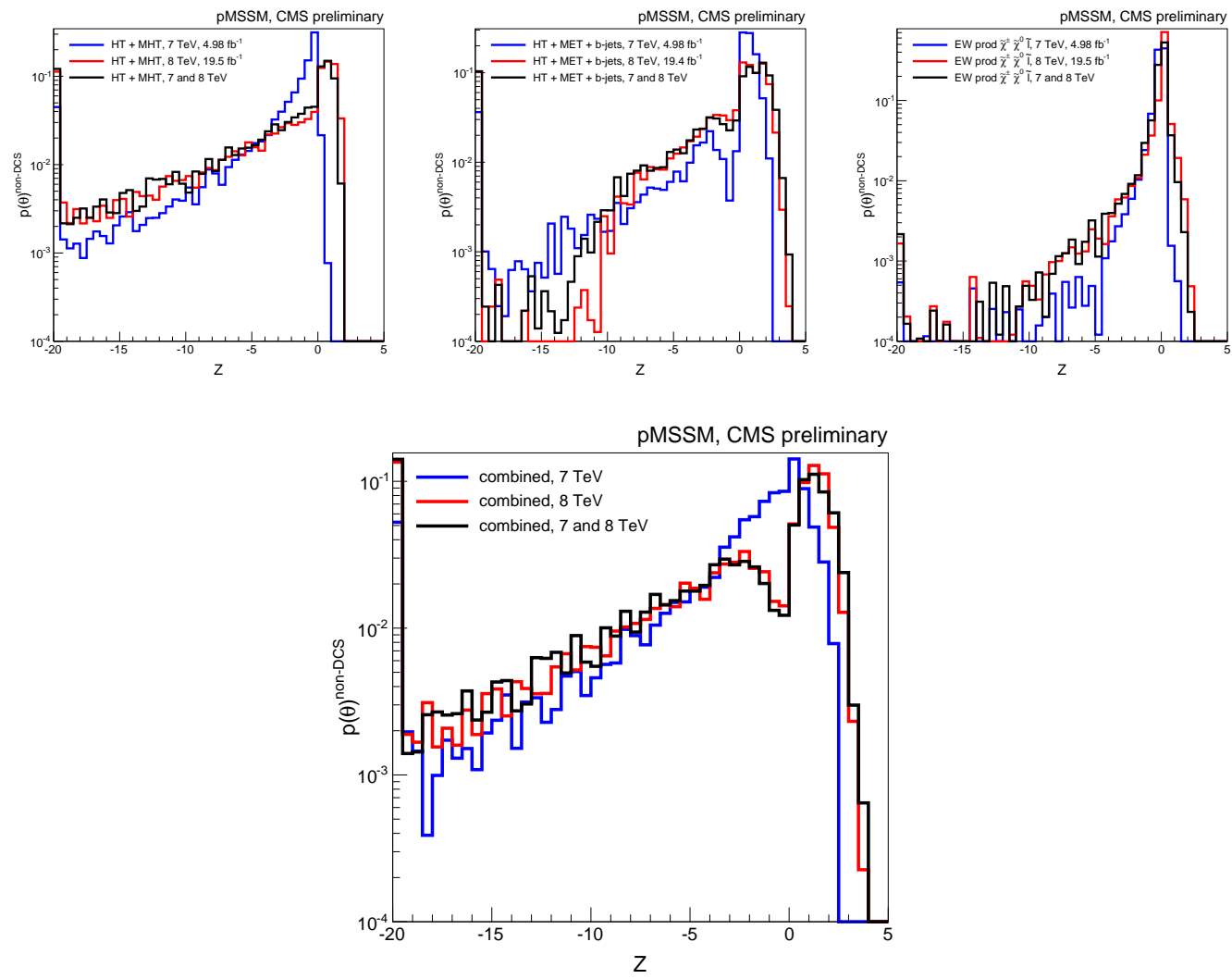

Figure 3: Distributions of $\mathrm{Z}$ for the CMS HT + MHT searches (top-left), the CMS HT + MET + b-jets searches (top-right), the CMS searches for EW production (bottom-left), and their combinations (bottom-right). Blue, red, and black line histograms show $\mathrm{Z}$ distributions including the data collected at respectively $7 \mathrm{TeV}, 8 \mathrm{TeV}$, and 7 and $8 \mathrm{TeV}$ [12].

can be useful in the next Run 2 of LHC, giving the opportunity to profit from both Run1 and Run2 data together to further explore the beyond Standard Model scenarios.

\section{References}

[1] ATLAS Collaboration, 2008 JINST 3 S08003.

[2] CMS Collaboration, 2008 JINST 3 S08004.

[3] ATLAS Collaboration, Phys.Lett. B716 (2012) 1-29, arXiv:1207.7214.

[4] CMS Collaboration, Phys.Lett. B716 (2012) 30-61, arXiv:1207.7235.

[5] J. Alwall, P. Schuster, and N. Toro, Phys.Rev. D79 (2009) 075020, arXiv:0810.3921.

[6] A. Djouadi et al., hep-ph/9901246.

[7] A. L. Read, J. Phys. G28 (2002) 2693.

[8] ATLAS Collaboration, JHEP 04 (2014) 169, arXiv:1207.7214.

[9] ATLAS Collaboration, JHEP 10 (2014) 96, arXiv:1407.0350. 
[10] ATLAS Collaboration, CERN-PH-EP-2015-138.

[11] ATLAS Collaboration, JHEP 11 (2014) 118, arXiv:1407.0583.

[12] CMS Collaboration, CMS-PAS-SUS-13-020. 